\title{
Can intraoperative ultrasound replace the frozen section in the assessment of ex vivo sentinel lymph node biopsy in early breast cancer in countries with limited resources?
}

Rasha Wessam Abdel Rahman ${ }^{1 *}$ (D), Emad Salaheldin Khallaf², Lamia Adel Salaheldin, Mohamed Nasr Hafez², Mohannad Aly Fayed ${ }^{2}$ and Somia Abdulatif Mahmoud Soliman²

\begin{abstract}
Background: Accurate staging and proper management of axillary lymph nodes (ALNs) in breast cancer patients are important for treatment. Surgical management of the axilla has evolved greatly in the last 20 years. Sentinel lymph node biopsy (SLNB), which was first investigated in the early 1990s, has replaced routine axillary lymph node dissection. This study evaluates the capability of using an ultrasound (US) as an alternative tool for the frozen section in the assessment of the ex vivo sentinel lymph node biopsy in countries with limited resources.

Results: The study is a prospective study that included 216 female patients with early breast cancer and negative axillary lymph nodes. All excised lymph nodes were examined by the intraoperative US and frozen section examinations. All the results were correlated with the final histopathological results. The number of negative nodes by US, frozen, and paraffin section examination was $58.30 \%, 69.40 \%$, and $69.40 \%$, respectively. The number of positive nodes by the US, frozen, and paraffin section examinations was $41.70 \%, 30.60 \%$, and $30.60 \%$ respectively. The sensitivity, specificity, PPV, NPV, and accuracy of US in the detection of positive lymph nodes were $95.45 \%, 82 \%$, $70 \%, 97.62 \%$, and $86.11 \%$, respectively, and the sensitivity, specificity, PPV, NPV, and accuracy of frozen examination in the detection of positive lymph nodes were $90.91 \%, 96 \%, 90.91 \%, 96 \%$, and $94.44 \%$, respectively.
\end{abstract}

Conclusion: Intraoperative US is a good negative test in the assessment of ex vivo SLNB, but it is not a good positive test, so it cannot replace the intraoperative frozen section in the assessment of SLNs.

Keywords: Ultrasonography, Sentinel lymph node biopsy, Frozen sections, Paraffin embedding

\section{Background}

Axillary lymph node (LN) metastasis is an important factor in the prognosis of breast cancer because of their impact on further patient management and overall survival. There are several pre-operative methods to detect metastases in the axillary lymph node. Some studies have used ultrasound (US) examination alone; others

\footnotetext{
* Correspondence: rashakao@yahoo.com

${ }^{1}$ Radiology Department, Faculty of Medicine, Cairo University, Giza, Egypt Full list of author information is available at the end of the article
}

have used fine-needle aspiration cytology (FNAC) with or without ultrasound guidance [1].

Sentinel lymph node biopsy (SLNB) is a minimally invasive technique for assessing the axilla; it had been used as an alternative to axillary lymph node dissection (ALND) and has become care management for clinically node-negative cancer patients as it is significantly reduced surgical morbidity [2].

Patients whose SLN have no or limited involvement (isolated tumor cells or micrometastases or macrometastases without extra-capsular extension) can omit ALND safely [3]. 
Immediate ALND with avoidance of second surgery for patients with tumor-positive axillary lymph nodes can be performed if intraoperative frozen pathological examination of SLN has been performed intraoperatively. No difference in local or distant recurrence rate has been reported in a prospective randomized ACOSOG Z0011 trial (of ALND vs. no further surgery for SLN-positive patients) who had ALND and those who did not. Although most patients in that trial had favorable clinical-pathological factors, the routine use of frozen section for the evaluation of SLNB is questionable [4].

However, qualified pathologist for frozen section assessment is not widely available. Hence, the search for another method to assess the SLNs intraoperatively is becoming very important.

We performed a 3-year prospective study to evaluate sensitivity, specificity, positive-negative predictive values, and accuracy of intraoperative ex vivo ultrasound examination of SLN biopsy specimens in comparison to intraoperative frozen section examination and final paraffin histopathological results to assess the value of intraoperative US in confirming positivity of SLNs.

\section{Methods}

\section{Case selection and data collection}

This prospective study was carried out between January 2015 and April 2018. The study was approved by the ethical committee of a multidisciplinary "Breast Cancer Hospital" and all enrolled patients provided their informed consent. We included 216 female breast cancer patients. The patient's ages ranged from 21 to 75 years (mean, 48.83years). Our inclusion criteria were early breast cancer patients (T1, T2 NOM0) with clinically and radiologically negative axillary lymph nodes who were candidates for SLNB. We excluded patients with positive axillary lymph nodes, post-neo-adjuvant chemotherapy, and those with contraindication for sentinel lymph node biopsy (e.g., inflammatory breast cancer).

\section{Surgical procedures}

A sentinel lymph node biopsy was performed by a specialized breast cancer surgeon who had experience in SLNB. After induction of anesthesia, injection of 2-3 ml of $1 \%$ methylene blue dye (prepared by using $50 \mathrm{mg}$ of MB powder diluted in $5 \mathrm{cc}$ saline) around the tumor or subareolar was performed, then after $15 \mathrm{~min}$, a small incision was done in the axilla $2 \mathrm{~cm}$ below the hairline near the anterior axillary fold, opening of axillary fascia, retrieval of 1-3 blue LN, any enlarged axillary LN, and the lymph nodes with bluish lymphatic streaks with good hemostasis was performed. The identification rate was $100 \%$ with no allergic manifestations reported at the time of surgery.

\section{Ex vivo intraoperative ultrasound examination}

The excised sentinel nodes were examined by a radiologist who had more than 10 years' experience in breast imaging, using a $12-\mathrm{MHz}$ linear-array ultrasound transducer after covering the probe with a rubber glove and applying plenty of gel over the examined node. The focus and depth of the machine were adjusted. The excised lymph nodes were examined regarding their size, shape, cortical symmetry (symmetrical or asymmetrical), cortical thickness, and preservation of their fatty hilae. Average 3-4 SLN were examined each time and the most suspicious lymph node with an asymmetrical cortical thickness or lost fatty hilum was requested to be the first one examined by the frozen section.

\section{Pathologic examination}

All lymph node specimens were examined by a dedicated breast pathologist by frozen section using toluidine blue staining. Each node was multi-sectioned and one represented section was processed for each node. The sections were cut at $1-2-\mathrm{mm}$ distance, then processed by an automated processor using different alcohol concentrations followed by xylene and paraffin wax. According to section results, further patient management occurred guided by ACOSOG Z0011 criteria as follows:

- Patients with negative SLNB: no further ALND

- Patients with ITCs (isolated tumor cells): no further ALND

- Patients with micrometastasis or macrometastasis (1-2 out of 3-4 positive lymph nodes): no further ALND

- Patients with 3 or more positive lymph nodes, matted lymph nodes, or lymph nodes with extracapsular extension: ALND was performed

Then, the SLN biopsy specimens were sent for paraffin examination. The paraffin blocks are serially cut at 4micron thickness stained by routine hematoxylin and eosin stains for pathological examination.

\section{Statistical analysis}

Data were coded and entered using the statistical package SPSS (Statistical Package for the Social Sciences) version 25. Data were summarized using mean, standard deviation, median, minimum, and maximum in quantitative data and using frequency (count) and relative frequency (percentage) for categorical data. Standard diagnostic indices including sensitivity, specificity, positive predictive value (PPV), negative predictive value (NPV), and diagnostic efficacy were calculated as described by [5]. ROC curve was constructed with an area under curve analysis performed to detect the best cutoff value of cortical thickness for the detection of + ve $\mathrm{LN}$. 
Table 1 The histopathological examination of the studied 216 cases

\begin{tabular}{lll}
\hline Histopathology & Number of cases & Percent \\
\hline Invasive ductal carcinoma & 183 & 84.7 \\
Invasive ductal carcinoma with in situ component & 6 & 2.8 \\
Mixed invasive lobular and invasive ductal carcinoma & 6 & 2.8 \\
Intra cystic papillary carcinoma & 6 & 2.8 \\
Paget disease & 6 & 2.8 \\
Ductal carcinoma in situ with microinvasion & 3 & 1.4 \\
Invasive ductal carcinoma with mucoid differentiation & 3 & 1.4 \\
Lobular carcinoma & 3 & 1.4 \\
\hline
\end{tabular}

Comparisons between quantitative variables were done using the non-parametric Mann-Whitney test [6]. For comparing categorical data, chi-square $\left(\chi^{2}\right)$ test was performed. An exact test was used instead when the expected frequency is less than 5 [7]. $P$ value of less than 0.05 was considered statistically significant.

\section{Results}

Two hundred and sixteen early breast cancer female patients T1-2, N0 M0 were a candidate for SLNB; their SLN specimens were examined intraoperatively by ultrasound. The long axis of the examined nodes ranged from 1.6 to $26 \mathrm{~mm}$ (mean $13.15 \mathrm{~mm}$ ) and their short axis ranged from 0.8 to $26 \mathrm{~mm}$ (mean $5.82 \mathrm{~mm}$ ). One hundred and fifty-six $(72.2 \%)$ patients had symmetrical nodal cortical thickness, $30(45.5 \%)$ of them were +ve and $126(84 \%)$ were -ve, while $60(27.8 \%)$ of the patients had asymmetrical diffuse cortical thickening, 36 (54.5\%) of them were +ve and 24 (16\%) were -ve. The nodal cortical thickness of the examined lymph nodes ranged from 0.9 to $8.6 \mathrm{~mm}$ (mean $2.59 \mathrm{~mm}$ ). The final histopathology results of our cases are demonstrated in Table 1. There was a statistically significant correlation between diffuse asymmetrical cortical thickness and paraffin results in the detection of positive lymph nodes (PV $<.001)$. There was also a statistically significant correlation between nodal cortical thickness and the paraffin results $(\mathrm{PV}<.001)$. The final positive lymph nodes by paraffin examination showed cortical thickness ranged from 1.4 to $8.6 \mathrm{~mm}$ in thickness (mean $=2.05 \mathrm{~mm}$ ) with cutoff value $=2.65 \mathrm{~mm}$. Using the cortical thickness in the detection of positive nodes showed an area under the curve $=0.857$ and $95 \%$ confidence interval $(0.798-$ 0.916 ) with $81.8 \%$ sensitivity and $80 \%$ specificity.

\section{The performance of the US in the evaluation of lymph node status}

The sensitivity, specificity, PPV, NPV, and accuracy of ultrasound in the detection of positive lymph nodes were $95.45 \%, 82 \%, 70 \%, 97.62 \%$, and $86.11 \%$, respectively (Table 2).

\section{The performance of the frozen section in the evaluation} of lymph node status

The sensitivity, specificity, PPV, NPV, and accuracy of frozen examination in the detection of positive lymph nodes were $90.91 \%, 96 \%, 90.91 \%, 96 \%$, and $94.44 \%$, respectively (Table 2).

\section{Discussion}

Giuliano et al. [8] are the first surgeons to introduce the technique of SLN for the assessment of nodal status in early node-negative breast cancer, and the technique has become a standard procedure for breast cancer patients. SLN biopsy had less morbidity compared with ALND and the success of the procedure depends on cooperation between the multidisciplinary team of specialist surgeons, pathologists, and nuclear medicine. As with any technique, the frozen section has its limitation such as cost, labor, a requirement of a skilled histopathology technician, and dedicated histopathologists [9].

So this study is performed to evaluate the performance of intraoperative US as a replacement tool for the frozen section in the ex vivo evaluation of axillary lymph node status in patients with early breast cancer in countries with limited resources and difficulties in establishing intraoperative frozen section for breast cancer patients.

Ultrasound examination of axillary LN has its characteristic features for suggesting malignant lymph node

Table 2 The performance of US and frozen section in the evaluation of axillary lymph node status

\begin{tabular}{llllll}
\hline & Sensitivity $(\mathbf{9 5} \% \mathrm{Cl})$ & Specificity $\mathbf{( 9 5 \% ~ C l )}$ & PPV (95\% Cl) & NPV (95\% Cl) & Accuracy (95\% Cl) \\
\hline Ultrasound & $\mathbf{9 5 . 4 5 \% ( 8 7 . 2 9 - 9 9 . 0 5 )}$ & $\mathbf{8 2 \% ( 7 4 . 9 0 - 8 7 . 7 9 )}$ & $\mathbf{7 0 \% ( 6 2 . 2 9 - 7 6 . 7 3 )}$ & $\mathbf{9 7 . 6 2 \% ( 9 3 . 1 2 - 9 9 . 2 0 )}$ & $\mathbf{8 6 . 1 1 \% ( 8 0 . 7 7 - 9 0 . 4 3 )}$ \\
Frozen & $\mathbf{9 0 . 9 1 \% ( 8 1 . 2 6 - 9 6 . 5 9 )}$ & $\mathbf{9 6 \% ( 9 1 . 5 0 - 9 8 . 5 2 )}$ & $\mathbf{9 0 . 9 1 \% ( 8 1 . 9 8 - 9 5 . 6 5 )}$ & $\mathbf{9 6 \% ( 9 1 . 7 9 \% - 9 8 . 1 )}$ & $\mathbf{9 4 . 4 \% ( 9 0 . 5 0 - 8 7 . 1 0 )}$ \\
\hline
\end{tabular}




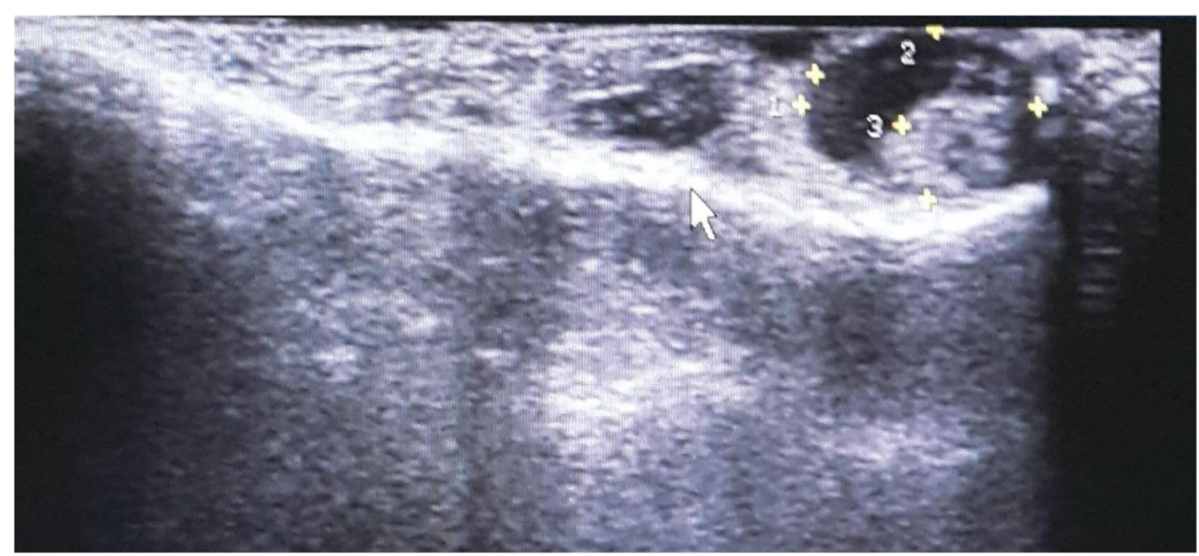

Fig. 1 US of ex vivo SLNB showing asymmetrical cortical nodal thickening reaching $3.2 \mathrm{~mm}$. It was considered positive by the US, and it was positive by frozen and paraffin examination

involvement and has been established as a pre-operative non-invasive tool to determine axillary LN status [10].

Further management of SLNB positive nodes was guided by ACOSOG Z0011 criteria for the management of SLNB in T1-2 N0 patients according to NCCN guidelines [11].

In the current study, SLNB was done using $1 \% \mathrm{MB}$ with a $100 \%$ identification rate (IR). It was found that identification rates for SLNB have increased over time from $88 \%$ in $1992-2000$ to $97 \%$ in $2007-2012$, the increase in IR during the last 18 years is likely due to the increase in gained experience by the surgeons performing SLNB [12].

In the ACOSOG Z0010 trial, the percent of failed SNB with blue dye was $1.4 \%$, radiocolloid $2.3 \%$, and the combination $1.2 \%$, so some authors claim that the addition

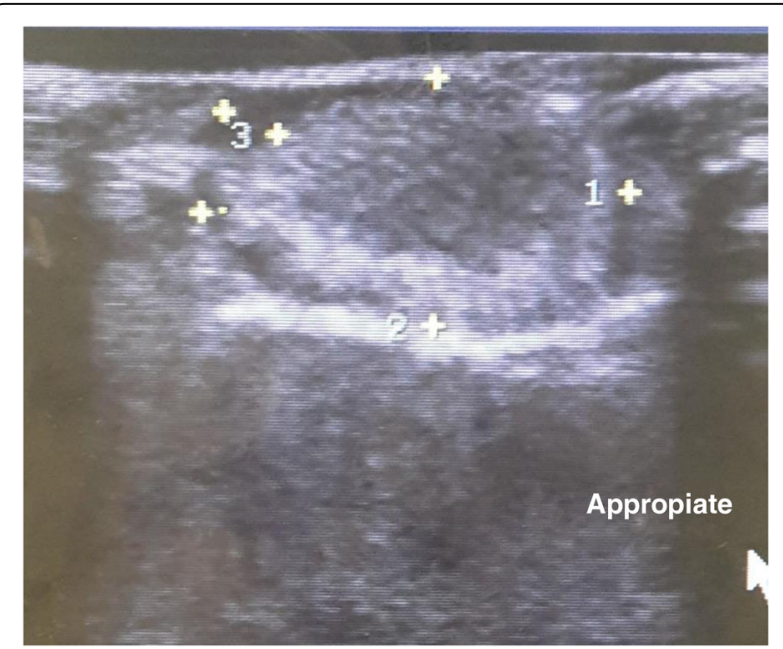

Fig. 2 US of ex vivo SLNB showing asymmetrical cortical nodal thickening reaching $2 \mathrm{~mm}$. It was considered positive by the US, and it was positive by frozen and paraffin examination of radiocolloids to blue dye in SLNB does not increase the identification rate to the degree that justifies its costs and restrictions in its use [13].

The commonly used technique for intraoperative detection of sentinel lymph node metastasis is a frozen section examination; its reported sensitivity range from 19 to $75 \%$ [14]. However, with increased experience, Wong et al. [15] reported the overall false-negative rate for the frozen section in SLN was 13.5\%. The authors considered this rate is acceptable.

In the current study, all lymph node specimens were examined by a dedicated pathologist; it was found that the sensitivity, specificity, PPV, NPV, and accuracy of frozen examination in the detection of positive lymph nodes were $90.91 \%, 96 \%, 90.91 \%, 96 \%$, and $94.44 \%$, respectively.

This study has shown significantly improved results which may be due to serial slicing of lymph nodes, examining multiple levels, and the proven expertise of the histopathologist.

In the current study, all lymph node specimens were examined by a dedicated pathologist; it was found that the sensitivity, specificity, PPV, NPV, and accuracy of frozen examination in the detection of positive lymph nodes were $90.91 \%, 96 \%, 90.91 \%, 96 \%$, and $94.44 \%$, respectively. This study has shown significantly improved results which may be due to serial slicing of lymph nodes, examining multiple levels, and the proven expertise of the histopathologist.

Our results were in agreement with Krag et al. [16], who reported $90.2 \%$ sensitivity of sentinel lymph node examination in breast cancer patients $9.8 \%$ false-negative results.

Also, Marano et al. [17] have retrospectively evaluated 359 sentinel node biopsies in breast cancer from January 2011 to December 2018, performing an intraoperative 


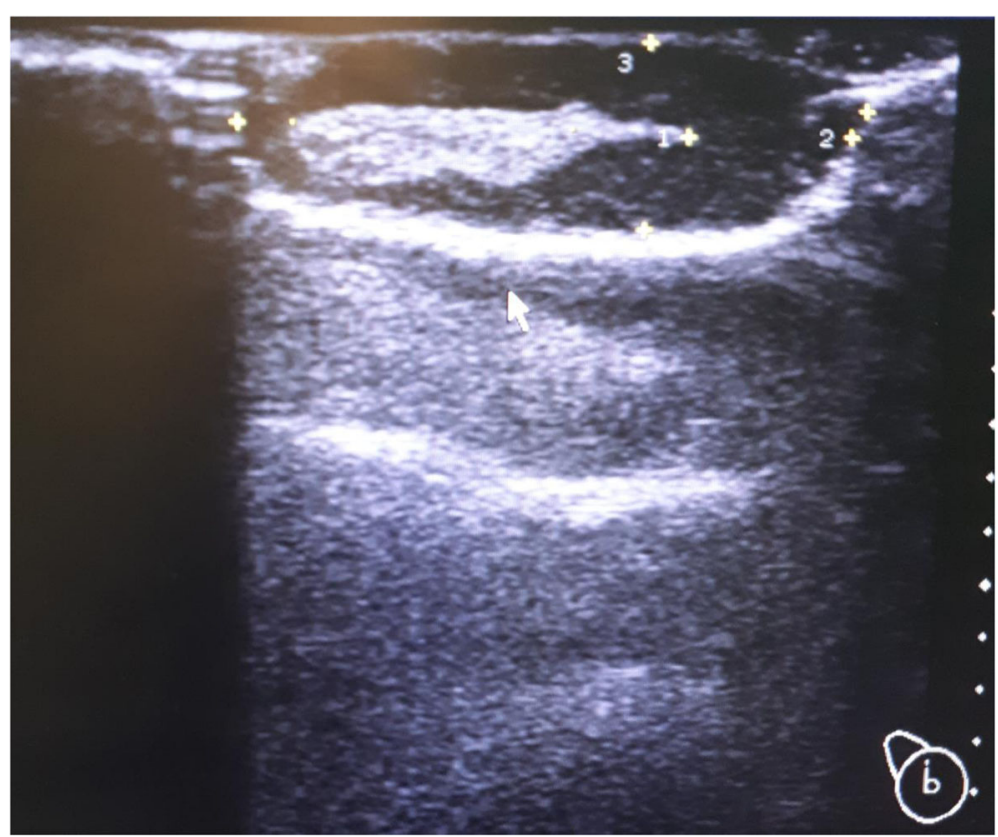

Fig. 3 US of ex vivo SLNB showing asymmetrical cortical nodal thickening reaching $6.5 \mathrm{~mm}$. It was considered positive by the US, and it was negative by frozen and paraffin examination

examination. It results in $12.8 \%$ "false negative" rate, in which only $4.2 \%$ in macrometastases, with an overall sensitivity of $68.4 \%$ (macrometastases, $86 \%$; micrometastases, $11 \%$ ), an overall specificity of $98.7 \%$ and an overall accuracy of $89.7 \%$.

In this study, we explored the role of another new technique by using intraoperative ultrasound for the evaluation of the ex vivo SLN before being examined by the frozen section during the operation and finally by the paraffin section postoperatively. It is a possible future technique where intraoperative ultrasound assessment of the ex vivo SLN detects the abnormal nodal architecture (cortical thickening $>3 \mathrm{ml}$, loss of the normal hyperechoic hilum). Cortical thickening is an early finding, with eccentric thickening having a higher predictive value for node involvement than diffuse thickening. A cutoff value of $3 \mathrm{~mm}$ for defining a thickened versus normal cortex is most common, but not

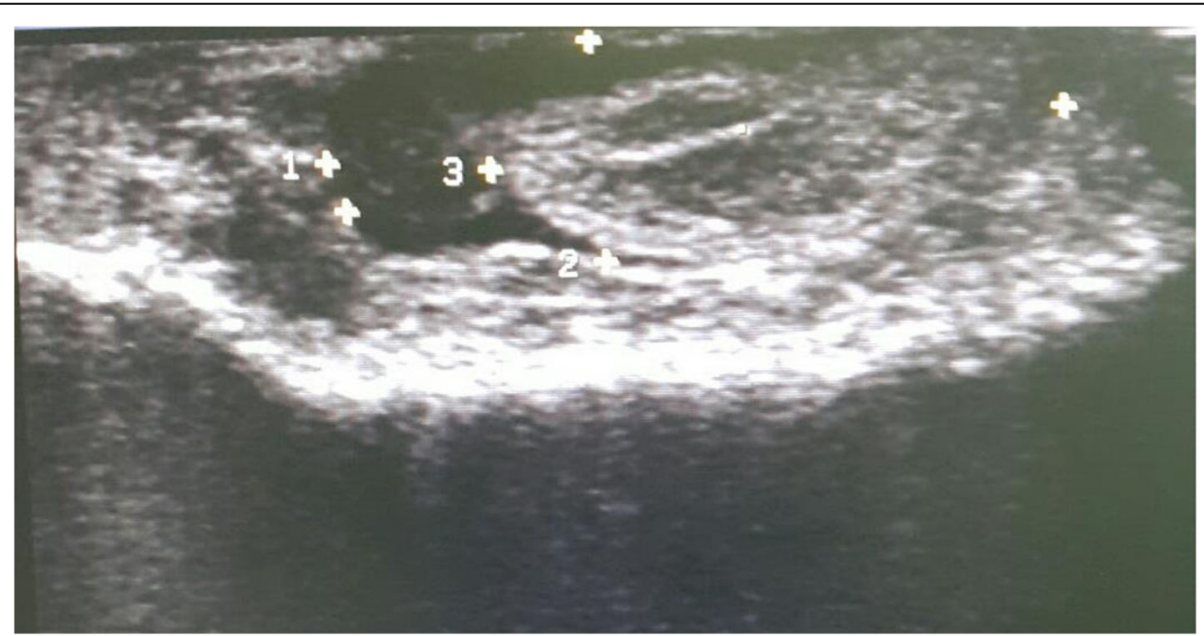

Fig. 4 US of ex vivo SLNB showing asymmetrical cortical nodal thickening reaching $3.7 \mathrm{~mm}$. It was considered positive by the US, and it was negative by frozen and paraffin examination 
universally, used. The presence of a tumor within the node may lead to the effacement of the fatty hilum and alteration in nodal contours [18].

Suspicious criteria for axillary LN in US evaluation include increase spherical index, nodal hypoechogenicity, and cortical thickness. Along with asymmetrical cortical thickening, an abnormal lymph node can be defined. Cortical thickness of $\geq 3 \mathrm{~mm}$ showed $96 \%$ sensitivity and $87 \%$ specificity in the detection of malignant nodes; other trials with a larger sample size used values ranging between 2.3 and $4 \mathrm{~mm}$ [18].

We categorized the retrieved lymph node as being suspicious depending on the measurement of its cortical thickness and whether it has a uniform or non-uniform cortical thickness (Figs. 1 and 2). Both used criteria showed a statically significant correlation with final histopathological results ( $p$-value $<0.001)$ with a cutoff value for cortical thickness $=2.65 \mathrm{~mm}$.

The sensitivity, specificity, PPV, NPV, and accuracy of ultrasound in the detection of positive lymph nodes were $95.45 \%, 82 \%, 70 \%, 97.62 \%$, and $86.11 \%$, respectively.

By comparing ultrasonographic assessment of ex vivo lymph nodes with frozen section examination to paraffin section examination of the lymph nodes, we found that frozen section examination is superior to the ultrasonographic assessment of ex vivo lymph in detecting positive cases when compared to paraffin section examination (Figs. 3 and 4).

In the current study, injection of $1 \% \mathrm{MB}$ was safe and no allergic or anaphylactic reactions were observed during the SLNB procedure, following most published studies, except Teknos et al. [19] who reported a case of pulmonary edema during an SLN procedure using MB.

Follow-up of the patients with positive SLNB for an average of 1 year showed no locoregional recurrence in any of the patients in the study group.

\section{Study limitation}

This study described the results of a single-center with a small number of studied populations.

\section{Conclusion}

Ultrasound assessment of ex vivo SLNB is a good negative test with a sensitivity reaching $95.5 \%$ but it is not a good positive test and it is inferior to frozen section examination in detecting positive cases of SLNB. This technique cannot replace the intraoperative frozen section pathological assessment of SLNs.

\section{Abbreviations}

LN: Lymph node; US: Ultrasound; SLNB: Sentinel lymph node biopsy; FNAC: Fine-needle aspiration cytology; ALND: Axillary lymph node dissection; ITCs: Isolated tumor cells; IR: Identification rate

\section{Authors' contributions}

RW and MA wrote the manuscript. MN collected patient data. LA contributed to the image processing and collection of patient's images. EK participated in the design of the study and performed the surgical procedure. SA performed the pathological examination. RW and LA participated in its design and coordination and helped to draft the manuscript. MA was responsible for the revision of the draft from a clinical point of view. MN and MA were responsible for the surgical data collection. The author(s) read and approved the final manuscript.

\section{Funding}

None (there have been no significant financial support)

\section{Availability of data and materials}

The datasets used and/or analyzed during the current study are available from the corresponding author on reasonable request.

\section{Declarations}

\section{Ethics approval and consent to participate}

The medical ethics were considered and respected. This research is approved by Local Ethical Committee of Kasr El Aini Medical School, Faculty of Medicine, Cairo University (reference number is not available), and informed written consent was taken from patients included in this study.

\section{Consent for publication}

All patients included in this research gave written informed consent to publish the data contained within this study.

\section{Competing interests}

The authors declare that they have no competing interests.

\section{Author details}

${ }^{1}$ Radiology Department, Faculty of Medicine, Cairo University, Giza, Egypt. ${ }^{2}$ Faculty of Medicine, Cairo University, Giza, Egypt.

Received: 29 October 2020 Accepted: 27 April 2021

Published online: 02 June 2021

\section{References}

1. Alkuwari E, Auger M (2008) Accuracy of fine-needle aspiration cytology of axillary lymph nodes in breast cancer patients. A study of 115 cases with cytologic -histologic correlation. Am Cancer Soc 114:89-93

2. Siguan SS, Bordeos LN, Baking-Fernandez STA (2016) A review of the accuracy of sentinel lymph node biopsy by comparing frozen section with final paraffin block H\&S staining and correlation with the final axillary lymph node dissection results. Philipp J Surg Specialists (PJSS) 71:41-45

3. Van der Noordaa MEM, Peeters V, Rutgers EJT (2017) The intraoperative assessment of sentinel nodes -standard and controversies. Breast 34:64-69

4. Giuliano AE, Hunt KK, Ballman KV, Beitsch PD, Whitworth PW et al (2011) Axillary dissection vs. no axillary dissection in women with invasive breast cancer and sentinel node metastasis: a randomized clinical trial. JAMA 9: 569-575

5. Galen RS (1980) Predictive values and efficiency of laboratory testing. Pediat J Clin North Am 27:861-869

6. Chan YH (2003) Biostatistics 102: quantitative data - parametric \& nonparametric tests. Singapore Med J 44(8):391-396

7. Chan YH (2003) Biostatistics 103: qualitative data -tests of independence. Singapore Med J 44(10):498-503

8. Giuliano AE, Kirgan DM, GuentherJM MDL (1994) Lymphatic mapping and sentinel lymphadenectomy for breast cancer. Ann Surg 220(3):391-401. https://doi.org/10.1097/00000658-199409000-00015

9. Moatasim A, Mujtaba S, Faidi N (2013) Intraoperative frozen section analysis of sentinel lymph nodes in breast carcinoma patients in a tertiary hospital in Pakistan. Int J Surg 1:253-258

10. Gurleyik G, Guleyik E, Aktekin A, Aker F (2015) Preoperative assessment of the axilla by surgeon performed ultrasound and cytology in patients with breast cancer. J Clin Med Res 7(6):440-445. https://doi.org/10.14740/ jocmr $2114 \mathrm{w}$

11. Daly MB, Pilarski R, Axilbund JE, Buys SS, Crawford B, Friedman S, Garber JE, Horton C, Kaklamani V, Klein C, Kohlmann W (2014) Genetic/familial high - 
risk assessment: breast and ovarian, version 1.2014. J Natl Compr Canc Netw 12(9):1326-1338. https://doi.org/10.6004/jnccn.2014.0127

12. Niebling MG, Pleijhuis RG, Bastiaannet E, Brouwers AH, Van Dam GM, Hoekstra HJ (2016) A systematic review and meta-analysis of sentinel lymph node identification in breast cancer and melanoma, a plea for tracer mapping. Eur J Surg Oncol 42(4):466-473. https://doi.org/10.1016/.jejso.201 5.12 .007

13. Hung WK, Chan CM, Ying M, Chong SF, Mak KL, Yip AW (2005) A randomized clinical trial comparing blue dye with combined dye and isotope for sentinel lymph node biopsy in breast cancer. Br J Surg 92(12): 1494-1497

14. Lombardi A, Maggi S, Stenzani G, Amanti Frozen C (2018) Frozen section in sentinel lymph node biopsy for breast cancer in the era of ACOSOG Z0011 and IBCSG 23-10 trials. Eur J Cancer 4(1):232-236

15. Wong J, Yong WS, Thicke AA, lqbal J, Salahuddin AS, Ho GH, Madhukumar P, Tan BK, Ong KW, Tan PH (2015) The false-negative rate for intraoperative sentinel lymph node frozen section in patients with breast cancer: a retrospective analysis of patients in a single Asian institution. J Clin Pathol 4(8):536-540

16. Krag DN, Anderson SJ, Julian TB, Brown AM, Harlow SP, Ashikaga T et al (2007) Technical outcomes of sentinel-lymph-node resection and conventional axillary-lymph-node dissection in patients with clinically nodenegative breast cancer: results from the NSABP B-32 randomised phase III trial. Lancet Oncol 8:881-888

17. Marano A, Sodano B, Vitiello C, Martini AM, Baldassarre F, Siciliano A, lannaci $G$ (2020) Sentinel lymph node biopsy with intraoperative touch imprint cytology $(\mathrm{TIC})$ in breast cancer: experience of a mild-volume center. G Chir 41(1):94-98

18. Oz A, Dermirkazik FB, Akpinar MG, Soygur I, Baykal A, Onder SC, Uner A (2012) The efficiency of ultrasound and ultrasound-guided fine-needle aspiration cytology in the preoperative assessment of axillary lymph node metastasis in breast cancer. J Breast Cancer 15(2):211-217. https://doi.org/1 0.4048/jbc.2012.15.2.211

19. Teknos D, Ramcharan A, Oluwole SF (2008) Pulmonary edema associated with methylene blue dye administration during sentinel lymph node biopsy. J Natl Med Assoc 100(12):1483-1484. https://doi.org/10.1016/S00279684(15)31552-2

\section{Publisher's Note}

Springer Nature remains neutral with regard to jurisdictional claims in published maps and institutional affiliations.

\section{Submit your manuscript to a SpringerOpen ${ }^{\circ}$ journal and benefit from:}

- Convenient online submission

- Rigorous peer review

- Open access: articles freely available online

- High visibility within the field

- Retaining the copyright to your article

Submit your next manuscript at $\boldsymbol{\nabla}$ springeropen.com 